\title{
Publisher Correction: Fizzy Fluxes
}

\section{Laura Zinke}

Correction to: Nature Reviews Earth \& Environment https://doi.org/10.1038/s43017-021-00205-4, published online 20 July 2021.

The use of mmol has now been corrected to Mmol in the HTML and PDF versions of the article.

https://doi.org/10.1038/s43017-021-00208-1 I Published online 28 July 2021

๑) Springer Nature Limited 2021 